\title{
Reliability of quantitative point shear-wave ultrasound elastography on vastus medialis muscle and quadriceps and patellar tendons
}

\author{
Enrico Maria Zardi ${ }^{1}$, Edoardo Franceschetti ${ }^{2}$, Chiara Giorgi ${ }^{3}$, Alessio Palumbo ${ }^{2}$, Francesco \\ Franceschi $^{2}$
}

${ }^{1}$ Hospitalist Service, "Campus Bio-Medico" University, Rome, ${ }^{2}$ Department Upper and Lower Limb Surgery Unit, University Campus Bio-Medico, Rome, ${ }^{3}$ Radiology Department, S. Maria della Misericordia Hospital, Urbino, Italy

\begin{abstract}
Aim: The aim of this work was to evaluate the reliability of pSWE in assessing the stiffness of the vastus medialis muscle and of the quadriceps and patellar tendons. Material and methods: For this purpose, 18 subjects (9 males and 9 females of $57 \pm 22$ years) in good clinical conditions were included in this study. pSWE examination was conducted by a unique expert operator with more than ten years of experience in musculoskeletal ultrasound. Two sets of five measurements for each muscle and tendon district were bilaterally performed at the same manner, at least fifteen minutes apart. The mean value of the measurements of each set was statistically compared with that of the other set. Results: No significant differences were found comparing the mean value of the measurements of the two sets of evaluation performed in muscle and tendon areas (vastus medialis muscle: $\mathrm{p}=0.285$; quadriceps tendon: $\mathrm{p}=0.979$; patellar tendon: $\mathrm{p}=0.187$ ). The intraclass correlation coefficient was excellent for all areas (vastus medialis muscle: 0.969; quadriceps and patellar tendons: 0.995 and 0.989, respectively). Conclusion: The pSWE technique demonstrated that it was a reliable method for measuring stiffness in vastus medialis muscle and quadriceps and patellar tendon in subjects who had undergone orthopedic surgery. This opens the possibility of many applications in monitoring stiffness before and after surgery and during rehabilitation.
\end{abstract}

Keywords: muscle; tendons; point shear wave elastography; stiffness

\section{Introduction}

The concept of elastography was initiated with the discovery that there is a direct proportionality between the absolute value of the strain of a tissue along the compression axis and the magnitude of the initial compression; the stress produced on the tissue causes in turn a measurable counter-stress by the compressed tissue itself.

Received 30.08.2018 Accepted 21.11.2018

Med Ultrason

2019, Vol. 21, No 1, 50-55

Corresponding author: Enrico Maria Zardi, MD, PhD

Hospitalist Service - Department Upper and Lower Limb Surgery Unit, Campus Bio-Medico University, Via Álvaro del Portillo 200 00128, Rome, Italy

e-mail: e.zardi@unicampus.it
That is why, more than 20 years ago, there was the first attempt to use the sonoelastographic method in the measurement of the viscoelastic properties of human muscles in situ [1]. Since then, much progress has been made and this technique was perfected and incorporated as a new application in the high-end sonographic machines. Strain elastography, that measures the modification in the length of the tissue during a continuous compression and decompression of the skin, was the first modality to have a potential use [2,3]. Shear wave elastography (SWE), which calculates shear Young's elastic modulus, via the measurement of the lateral propagation velocity of the shear waves after an ultrasound focused beam generated by the transducer, will be coming at a later date [4] and is still being developed for clinical use.

Thanks to the fact that these techniques provide respectively semi-quantitative and quantitative elasticity 
parameters, they have gained interest for applications in a number of areas such as liver, breast, thyroid and in musculoskeletal system [5-9].

Compared to strain elastography, SWE has the advantage that it needs no manual compression for stiffness measurements. Furthermore, while strain elastography provides measurements of the strain of a tissue area with respect to a surrounding one, or the strain ratio of two manually selected regions of interest (ROIs) [10], SWE may give a precise and accurate estimation of the stiffness in a determinate area inside the box of the measurement, giving us the result in velocity (meter per second) or in kilopascal $(\mathrm{kPa})$. This, besides simplifying the possibility to double-check after a certain time, also facilitates the use of SWE because it helps reduce the forecasting sample errors.

Despite a growing interest in studies on the utility of SWE in evaluating the stiffness in different tissues in many medical conditions, with a few exceptions [11], there is a paucity of information on the repeatability in the musculoskeletal system in healthy subjects, an absolutely necessary condition to accept the results of this technique. Therefore, the aim of this work is to evaluate whether, in the musculoskeletal application, SWE can be shown to be a reliable method.

\section{Materials and methods}

\section{Patients}

We planned an observational prospective study on the reliability of stiffness measurements in the musculoskeletal system, carrying out two sets of evaluations at least fifteen minutes apart, each of which consisted of five measurements. To this purpose, point-SWE (pSWE) was bilaterally performed on the vastus medialis muscle and on the quadriceps and patellar tendons of eighteen consecutive subjects in good clinical conditions (with no illness) referred to the Orthopedic Unit of this University for orthopedic surgical treatment not related to the studied structures. Each subject gave written consent to participate in this study which had received the approval of the Ethics Committee of the University.

\section{Ultrasound examination}

The examination was conducted by a single experienced sonographer, with more than ten years of experience in musculoskeletal ultrasound. A MyLab nine Esaote sonographic machine, equipped with a pSWE (QElaXto ${ }^{\circledR}$ system) and 4-15 MHz linear probe was used. This pSWE system allows the generation of an ultrasound focused beam that produces a localized perturbation in a single point of the tissue causing a shear wave response. The pSWE system reads and quantifies the fol- lowing propagation velocity of the shear waves generated, transforming it in KPa values through the Young's Modulus formula, with the assumption that the tissue's elastic response is linear and the tissue density is always $1000 \mathrm{~kg} \mathrm{~m}^{-3}[12,13]$.

Each patient was placed in a relaxed supine position without passive movements. To optimize the ultrasonographic images, a preset musculoskeletal examination setting was adopted, regulating gain and depth according to the patient's anatomy and being careful to visualize well the edges of muscles and tendons by maintaining the ultrasound beam position perpendicular to their fibers. Attention was also given to avoid abnormal compression, minimizing the pressure exerted by the probe on the tissues. The box size and shape of the pSWE were constant for all measurements.

The pSWE examination was performed bilaterally in three areas: a) vastus medialis muscle, in the distal third, in longitudinal view according to previous published studies $[11,14]$, with the legs out straight, slightly parted (the knee positioned at about $15^{\circ}$ of flexion). The box was placed $1 \mathrm{~cm}$ from the myotendinous junction to a depth of little more than $0.5 \mathrm{~cm}$ from the skin surface (fig 1); b) quadriceps tendon, scanned in longitudinal view with the knees slightly bent; the box was placed in the distal third of the tendon making sure it was far away from the bone (fig 2); c) patellar tendon, scanned in longitudinal view with the knees slightly bent; the box was placed to the exact half of the tendon (fig 3).

The mean value of five bilateral measurements for each patient in the two sets of evaluations, performed fifteen minutes apart, was used for the statistical comparison. The mean time spent to perform the pSWE examination was about $10 \pm 1$ minute in the first set of evaluations and $9 \pm 1$ minute in the second set.

\section{Statistical analysis}

Clinical data were collected on a Microsoft Excel version 2013 for Windows and were expressed as the mean \pm standard deviation (SD). A graphpad Software SPSS ${ }^{\circledR}$ (Statistical Package for Social Studies) version 24.0 (IBM Corporation, Chicago, IL, USA) was used to analyze the results. Shapiro-Wilk test was adopted to evaluate whether the data were normally or non-normally distributed. Coefficient of variation was also calculated.

The Wilcoxon signed rank test and two tail paired $t$ Test were respectively performed for non-parametric and parametric values to analyse whether there were differences between the first set and the second set of results. A $p$ value $<0.05$ was considered statistically significant (considering alpha error to be 5\%). Finally, intraclass correlation coefficient (ICC) was adopted to evaluate the 


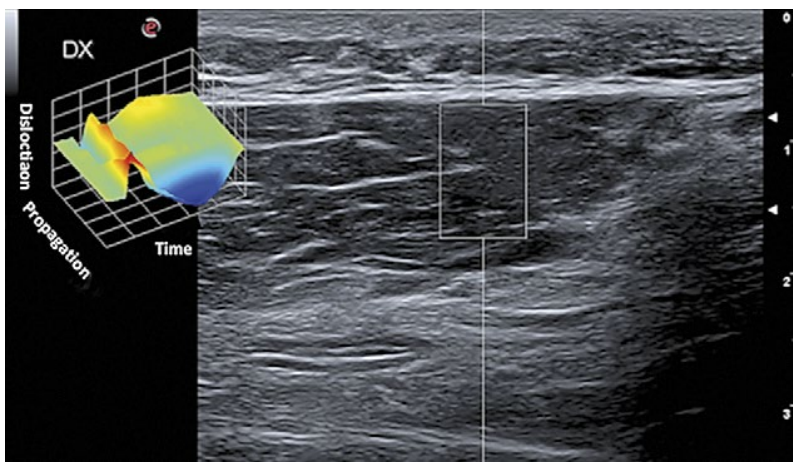

Fig 1. Point shear wave elastography (pSWE) of the vastus medialis muscle along the longitudinal axis. The ROI corresponds to the box area placed $0.7-1.7 \mathrm{~cm}$ from the skin.

repeatability of the pSWE comparing the results obtained in the first set of evaluation with those in the second set [15]. In accordance with other authors, the interpretation of the ICC results was fixed as follows: poor agreement $(0.00-0.39)$, fair agreement $(0.40-0.59)$, good agreement $(0.60-0.74)$ and excellent agreement $(0.75-1.00)$ $[16,17]$.

\section{Results}

The study population was represented by 18 subjects ( 9 men and 9 women, mean age $57 \pm 21$ years with a body mass index of $23.4 \pm 2.8$ ), 15 of which underwent surgical treatment of the knee (prosthesis or meniscectomy), 1 foot surgery and 2 shoulder instability correction. All examinations were performed before the surgical intervention. The Shapiro-Wilk test, performed both for the first and the second set of data, showed non-normal distribution of the pSWE values of the vastus medialis and normal distribution for those of the quadriceps and patellar tendons (Table I and fig 4).

\section{Reliability measurements}

First and second set of pSWE evaluations showed the following coefficient of variation: vastus medialis muscle (0.56 and 0.53$)$, quadriceps tendon ( 0.527 and 0.528$)$, patellar tendon (0.623 and 0.629). No significant differences were present regarding the stiffness of the vastus medialis muscle, quadriceps and patellar tendons comparing the first set of pSWE evaluations with the second set, performed at least fifteen minutes apart $(p=0.285$; $\mathrm{p}=0.97 ; \mathrm{p}=0.187$ ) (fig 5).

The ICC of pSWE evaluations showed excellent agreement for all areas examined: vastus medialis muscle 0.969 [95\% CI $(0.939 ; 0.984)]$; quadriceps tendon 0.995 [95\% CI $(0.990 ; 0.998)]$; patellar tendon 0.989 [95\% CI $(0.978 ; 0.995)]$.

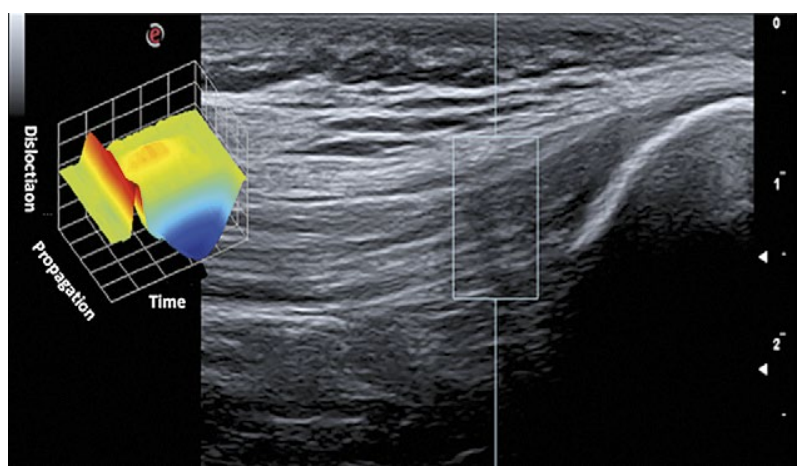

Fig 2. Point shear wave elastography (pSWE) of the quadriceps tendon along the longitudinal axis. The ROI corresponds to the box area placed $0.8-1.8 \mathrm{~cm}$ from the skin.

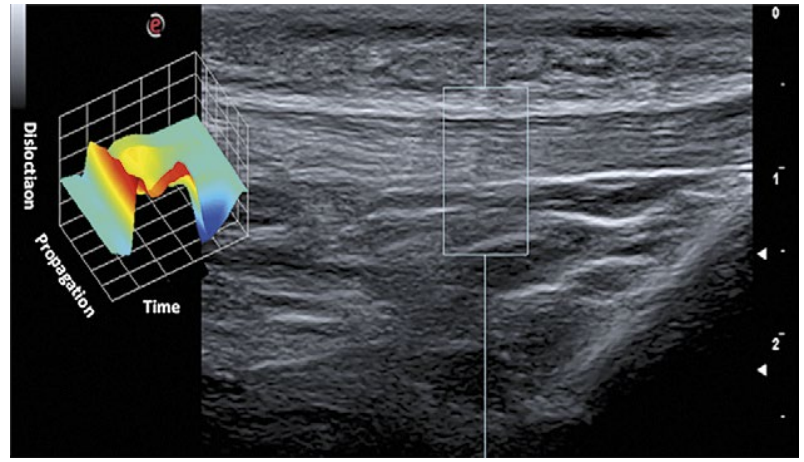

Fig 3. Point shear wave elastography (pSWE) of the patellar tendon along the longitudinal axis. The ROI corresponds to the box area placed $0.5-1.5 \mathrm{~cm}$ from the skin.

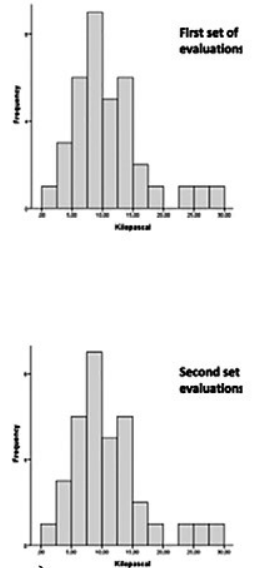

a)
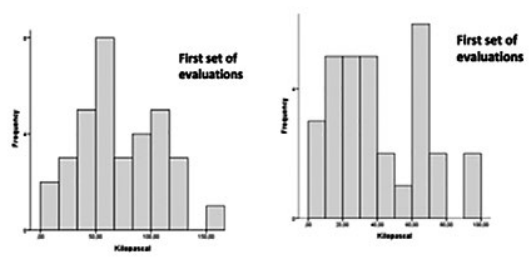

Fig 4. The histograms show the distribution of stiffnes values of vastus medialis muscle (Panel A), quadriceps (Panel B) and patellar (Panel C) tendons from 18 subjects. The Shapiro Wilk normality test confirmed a normal distribution of values for quadriceps and patellar tendons and a non-normal for vastus medialis muscle. 
Table I. Shapiro Wilk test of pSWE data in kilopascal (kPa) subdivided in two sets of evaluations

\begin{tabular}{|c|c|c|c|c|c|c|c|}
\hline & Mean value & SD & $95 \% \mathrm{CI}$ & Median & Asimmetry & Kurtosis & p value \\
\hline & \multicolumn{7}{|c|}{ pSWE first set of evaluations } \\
\hline Vastus medialis & 11.27 & 6.3 & {$[9.1 ; 13.4]$} & 9.9 & 1.239 & 1.820 & 0.006 \\
\hline Quadriceps tendon & 72.60 & 38.31 & {$[59.23 ; 85.9]$} & 66.0 & 0.24 & -0.495 & 0.63 \\
\hline \multirow[t]{2}{*}{ Patellar tendon } & 40.3 & 25.1 & {$[31.1 ; 49.5]$} & 33.8 & 0.49 & -0.56 & 0.258 \\
\hline & \multicolumn{7}{|c|}{ pSWE second set of evaluations } \\
\hline Vastus medialis & 10.9 & 5.8 & {$[9.0 ; 12.9]$} & 9.7 & 1.216 & 1.661 & 0.007 \\
\hline Quadriceps tendon & 72.63 & 38.3 & {$[59.25 ; 86.0]$} & 71.1 & 0.179 & -0.66 & 0.76 \\
\hline Patellar tendon & 41.6 & 26.2 & {$[32.0 ; 51.2]$} & 34.4 & 0.57 & -0.34 & 0.11 \\
\hline
\end{tabular}

CI: confidence interval; pSWE: point shear wave elastography; SD: standard deviation

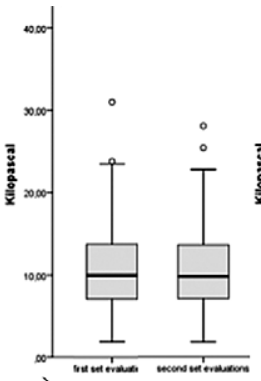

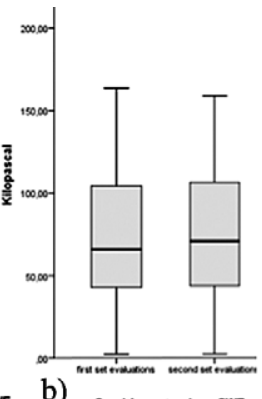

b) Ousaricops tondon pSWe

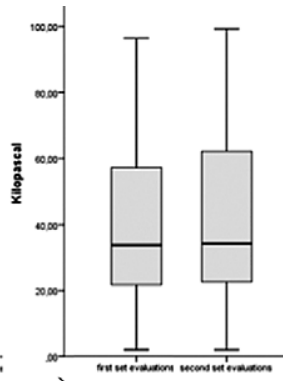

c) Patollar tondon pswe
Fig 5. The figure is subdivided in three Panels; in each Panel, the two box plots indicate the pSWE distribution obtained in two distinct set of evaluations performed at different time periods.

\section{Discussions}

The possibility of quantitatively measuring stiffness, by means of a sonographic software application, is an exciting prospect that is spreading in hospital practise, relying on the statement that the propagation velocity of the shear waves correlates with the elasticity of the tissues, increasing when it meets harder tissues. However, to avoid clinical malpractice, before encouraging a wider use of this method, it would be important to verify its reliability and robustness.

For this reason, we planned this study where the objective was to evaluate the reliability of pSWE method in muscle ant tendon evaluation. To this purpose, we aimed to measure the stiffness in three musculoskeletal areas: those of the vastus medialis muscle and of quadriceps and patellar tendons. In order to enlarge the variability of the test conditions, the patients selected for this study came from a wide range of ages and were of either sex. To make sure of performing a true test of repeatability, pSWE evaluations were conducted with the same modality in a longitudinal plane and at short intervals of time from each other, in all patients. Thus, the possibility of changes over time in stiffness properties, linked to the presence of some variables (hormonal variations, stress, trauma or other) was minimized and the possibility of forecasting sample errors due to different technical settings and positions was reduced [18]. Furthermore, in each patient only the average of five consecutive pSWE measurements of all muscle and tendon areas, was considered both in the first and in the second set of evaluation for the statistical analysis.

Following these adjustments, an excellent agreement was found between the results obtained in the first and in the second set of evaluations in each muscle and tendon areas. Vastus medialis muscle demonstrated to have stiffness values lower than those detected for tendons, in accordance with previous published data $[19,20]$.

The time spent to perform the first and the second set of evaluations was acceptable and similar for each patient, in both cases, thus being able to assume that this test is feasible.

The fact that there was a wide variation of values between patients was probably linked to the lack of a selective patient population. However, this did not affect the outcome; instead it made it more interesting for the study purpose. Indeed, the achievement of an excellent agreement between pSWE measurements in patients with different levels of muscle and tendon stiffness, makes this method available and useful in the follow up of any type of patients.

All of this opens the way to monitor the level of the stiffness of tendons and muscles after traumatic events or other solicitations such as orthopedic surgery, during rehabilitation or after sport events, as is starting to become customary [21]. It would be advisable to establish a range of normal value for any muscle and tendon areas grouped by age. In this manner, it would be easy to recognize immediately any deviation from normal values.

Obviously, the use of pSWE has some advantages and disadvantages $[22,23]$; it is a non-invasive cheap method, simple to apply and a more acceptable alternative for patients who have difficulty with magnetic reso- 
nance imaging that, at the same time, is also more expensive. The ability to see in real time in the monitor a shear wave graph, gives us a prompt feedback on the quality of the measurements, permitting any rapid corrections. Furthermore, there is an automatic reliability instrument that controls all measurements by referring to a statistical analysis of acquired radiofrequency signals; this tool accepts the values which are consistent and skips those off the charts. A disadvantage is surely the yet great variability of the values obtained that gives us no other choice than that of replicating measurements for each muscle and tendon area thus extending the time of investigation.

However, we point out that the real delay is in the order of five seconds for any additional measurement, namely a reasonable time-frame. Another disadvantage is the fact that the dimension of the box of the pSWE is fixed and cannot be adapted as necessary.

Some limitations affect this study: first, the low number of patients, although the examination was conducted bilaterally, thus doubling the measurements; second, the very short time laps between the first and the second sets of evaluations that did not permit to give any information on the repeatability of the method over a longer time period.

In conclusion pSWE is an easy tool to use that allows reproducible measurements of stiffness of vastus medialis muscle and quadriceps and patellar tendons, as proven by the result of excellent ICC values. Due to these results, we believe that pSWE is a reliable method and may be available for clinical use in the hospital practice for stiffness evaluation of these muscle and tendon areas.

\section{Conflicts of interests: none}

\section{References}

1. Levinson SF, Shinagawa M, Sato T. Sonoelastic determination of human skeletal muscle elasticity. J Biomech 1995;28:1145-1154.

2. Ophir J, Moriya T, Yazdi Y. A single transducer transaxial compression technique for the estimation of sound speed in biological tissues. Ultrason Imaging 1991;13:269-279.

3. Ophir J, Céspedes I, Ponnekanti H, Yazdi Y, Li X. Elastography: a quantitative method for imaging the elasticity of biological tissues. Ultrason Imaging 1991;13:111-134.

4. Wang H, Prendiville PL, McDonnell PJ, Chang WV. An ultrasonic technique for the measurement of the elastic moduli of human cornea. J Biomech 1996;29:1633-1636.

5. Kudo M, Shiina T, Moriyasu F, et al. JSUM ultrasound elastography practice guidelines: liver. J Med Ultrason (2001) 2013;40:325-357.

6. Barr RG, Zhang Z. Shear-wave elastography of the breast: value of a quality measure and comparison with strain elastography. Radiology 2015;275:45-53.
7. Hu X, Liu Y, Qian L. Diagnostic potential of real-time elastography (RTE) and shear wave elastography (SWE) to differentiate benign and malignant thyroid nodules: A systematic review and meta-analysis. Medicine (Baltimore) 2017;96:e8282.

8. Taljanovic MS, Gimber LH, Becker GW, et al. Shear-Wave Elastography: Basic Physics and Musculoskeletal Applications. Radiographics 2017;37:855-870.

9. Seliger G, Chaoui K, Kunze C, et al. Intra- and inter-observer variation and accuracy using different shear wave elastography methods to assess circumscribed objects - a phantom study. Med Ultrason 2017;19:357-365.

10. Carlsen JF, Ewertsen C, Lönn L, Nielsen MB. Strain Elastography Ultrasound: An Overview with Emphasis on Breast Cancer Diagnosis. Diagnostics (Basel) 2013;3:117-125.

11. Akkoc O, Caliskan E, Bayramoglu Z. Effects of passive muscle stiffness measured by Shear Wave Elastography, muscle thickness, and body mass index on athletic performance in adolescent female basketball players. Med Ultrason 2018;20:170-176.

12. Bercoff J, Tanter M, Fink M. Supersonic shear imaging: a new technique for soft tissue elasticity mapping. IEEE Trans Ultrason Ferroelect Freq Contr 2004;51:396-409.

13. Catheline S, Gennisson JL, Delon G, et al. Measuring of viscoelastic properties of homogeneous soft solid using transient elastography: An inverse problem approach. J Acoust Soc Am 2004;116:3734-3741.

14. Alfuraih AM, O'Connor P, Tan AL, Hensor E, Emery P, Wakefield RJ. An investigation into the variability between different shear wave elastography systems in muscle. Med Ultrason 2017;19:392-400.

15. Hripcsak G, Heitjan DF. Measuring agreement in medical informatics reliability studies. J Biomed Inform 2002;35:99-110.

16. Cicchetti DV, Sparrow SA. Developing criteria for establishing interrater reliability of specific items: applications to assessment of adaptive behavior. Am J Ment Defic 1981;86:127-137.

17. Fleiss JL. In: Statistical methods for rates and proportions. 2nd ed. New York: Wiley; 1981:212-236.

18. Kot BC, Zhang ZJ, Lee AW, Leung VY, Fu SN. Elastic modulus of muscle and tendon with shear wave ultrasound elastography: variations with different technical settings. PLoS One 2012;7:e44348.

19. Ewertsen C, Carlsen JF, Christiansen IR, Jensen JA, Nielsen MB. Evaluation of healthy muscle tissue by strain and shear wave elastography - Dependency on depth and ROI position in relation to underlying bone. Ultrasonics 2016;71:127-133.

20. Taş S, Onur MR, Yılmaz S, Soylu AR, Korkusuz F. Shear Wave Elastography Is a Reliable and Repeatable Method for Measuring the Elastic Modulus of the Rectus Femoris Muscle and Patellar Tendon. J Ultrasound Med 2017;36:565570 .

21. Andonian P, Viallon M, Le Goff C, et al. Shear-Wave Elastography Assessments of Quadriceps Stiffness Changes prior to, during and after Prolonged Exercise: A Longitudinal 
Study during an Extreme Mountain Ultra-Marathon. PLoS One 2016;11:e0161855.

22. Payne C, Watt P, Cercignani M, Webborn N. Reproducibility of shear wave elastography measures of the Achilles tendon. Skeletal Radiol 2018 47:779-784.
23. Baumer TG, Davis L, Dischler J, et al. Shear wave elastography of the supraspinatus muscle and tendon: Repeatability and preliminary findings. J Biomech 2017;53:201204. 